\title{
Caracterización sensorial de una hamburguesa precocida-congelada, usando carne caprina, perejil (Petroselinum crispum) y albahaca (Ocimum basilicum)
}

\author{
Sensory characterization of a precooked-frozen \\ hamburger using goat meat, parsley (Petroselinum \\ crispum) and basil (Ocimum basilicum)
}

Raúl Díaz Torres Universidad de Guayaquil (Guayaquil - Ecuador) raul.diazt@ug.edu.ec

Leila Águeda Prias Mogro Universidad de Guayaquil (Guayaquil - Ecuador)

Cynthia Mera Morales Universidad de Guayaquil (Guayaquil - Ecuador)

Revista Cumbres Vol.3 №2

Versión impresa ISSN 1390-9541

Versión electrónica ISSN 1390-3365

http://investigacion.utmachala.edu.ec/revistas/index.php/Cumbres 


\section{RESUMEN}

La metodología CHECK-ALL-THAT APPLY (CATA) es un método simple de obtener información sobre la percepción hedónica de los consumidores. El objetivo fue identificar, mediante metodología CATA, los atributos que podrían emplearse para optimizar formulaciones de hamburguesas con compuestos que aumenten su funcionalidad. Se utilizó un diseño factorial (2 factores con 3 niveles) para definir 9 formulaciones de hamburguesa de carne caprina, con diferentes niveles de adición de perejil (Petroselinum crispum) y albahaca (Ocimum basilicum) evaluándolas organolépticamente utilizando 80 consumidores potenciales, que identificaron los atributos presentes en cada formulación. Los resultados fueron procesados mediante el paquete estadístico SPSS. La validez interna (alfa de Crombach) fue 0,81 , indicando que las respuestas fueron consistentes. Los atributos Dureza, Sabor intenso, Jugosidad, Regusto, Consistencia y Picante son los que establecen las diferencias sensoriales entre fórmulas. Para los atributos señalados, la percepción fue significativamente notable, excepto para picante, importante para muy pocos jueces. La detección de sabor intenso y sabor picante fue mayor en fórmulas con menor condimentación. Esto puede indicar que los consumidores en realidad detectaban el sabor característico de la carne caprina, el cual se enmascara parcialmente por la acción de los condimentos empleados. En todas las formulaciones, los jueces declararon regusto, lo que puede asociarse al sabor característico de la carne caprina. En general los jueces consideraron que la dureza, consistencia y jugosidad, son detectables en todas las formulaciones. Los atributos dureza, sabor intenso, jugosidad, regusto, consistencia y picante son los considerados diferenciantes entre formulaciones.

Palabras clave: CATA, percepción sensorial, hamburguesa, carne caprina.

\section{ABSTRACT}

Using the CHECK-ALL-THAT APPLY methodology (CATA) is a simple method of obtaining information about the hedonic consumer perception and the reasons on which it is based. The aim of this work was to identify, using CATA methodology, those attributes that could be used for further optimization of burgers formulations with compounds that enhance their functionality. A factorial design (2 factors with 3 levels each) was used to define nine formulations of hamburgers based on goat meat, with the addition of parsley (Petroselinum crispum) and basil (Ocimum basilicum) in different levels. The organoleptic characteristics were evaluated using 80 potential consumers, which identified the attributes they considered were present in each of the formulations. The results were processed using the SPSS statistical software. The internal validity assessed by Cronbach's alpha was 0.81, indicating that responses were consistent. Hardness, Intense Flavor, Juiciness, Aftertaste, Consistency and Spiciness attributes proved to be those that set the sensory differences between the formulas. For all the attributes mentioned, the perception was significantly remarkable, except for the Spiciness attri- 
bute, which was important for very few participants. Detection of intense flavor and spiciness was greater for formulas with less presence of parsley and relatively low presence of basil. This may indicate that consumers actually detected the characteristic taste of goat meat, which in other formulas is partially masked by the action of seasonings used. In all formulations, the judges declared the presence of aftertaste, which may also be associated with the characteristic taste of goat meat. In general the participants considered that the hardness, consistency and juiciness, attributes are detectable in all formulations. The attributes hardness, intense flavor, juiciness, aftertaste, consistency, and spiciness are those that consumers detected as differentiating between formulations.

Keywords: CATA, sensory perception, hamburgers, goat meat.

\section{INTRODUCCIÓN}

El desarrollo de nuevos productos, que siendo innovadores aporten un valor agregado, se ha convertido en la estrategia para sobrevivir y lograr el éxito en el mercado global de alimentos, donde hay tres fuerzas que empujan la creación de nuevos productos: la elaboración de productos saludables, el deseo de consumir nuevos productos (como los de cocinas étnicas) y, por último, el desarrollo de productos de alta escala (Moskowitz \& Hartmann, 2008).

La evaluación sensorial de los alimentos constituye hoy en día un pilar fundamental para el diseño y desarrollo de nuevos productos alimenticios (Aguilar, Castillo \& Gutowski, 2014). Sin embargo, los métodos descriptivos tradicionales conllevan un consumo de recursos y tiempo muy elevado. Por lo tanto, en la industria alimentaria existe una creciente aceptación de métodos sensoriales alternativos, considerados más rápidos y rentables que los análisis descriptivos clásicos convencionales (Alegre et al., 2015).

Por décadas, la caracterización sensorial de los alimentos ha sido llevada a cabo mediante el análisis descriptivo, con panelistas altamente entrenados (Lawless \& Heymann, 2010), lo que implica la selección, entrenamiento y mantenimiento de un grupo de entre 8 y 20 asesores que evalúan la intensidad de los atributos característicos de cada producto (Heymann, King \& Hopfer, 2014). Estudios recientes muestran la posibilidad de utilizar a los consumidores para obtener directamente la información requerida (Antúnez et al, 2016).

La implementación de un sistema de análisis orientado al consumidor ha sido reconocida como la mejor vía para alcanzar el éxito en el desarrollo de nuevos productos (Grunert \& van Trijp, 2014). Para la industria de alimentos, un paso esencial en el desarrollo de nuevos alimentos es identificar una formulación lo más cercana posible a las preferencias sensoriales del consumidor (Bruzzone et al., 2015). La descripción sensorial de un producto por parte de los consumidores puede correlacionarse con los resultados de preferencia para intentar describir las características sensoriales del producto ideal (Moskowitz \& Hartmann, 2008).

Para lograr esta descripción del producto ideal pueden emplearse dife- 
rentes técnicas de análisis sensorial tales como el mapeo de preferencias o la comparación con productos líderes del mercado empleados como referencia en las encuestas al consumidor (Bruzzone et al., 2015), así como el Método del Perfil Ideal (MPI) en el cual a los consumidores no solo se les pregunta acerca de la intensidad percibida para cada atributo, sino también cual es la intensidad ideal deseable para dicho atributo (Worch \& Punter, 2014). El Método del Perfil Ideal ha sido bien estudiado en los últimos años y se ha demostrado su utilidad tanto para el desarrollo de nuevos productos como para la reformulación de los existentes (Worch, Lê, Punter \& Pagés, 2012), pero el uso de escalas de intensidad puede resultar tedioso para los consumidores (Ares, Bruzzone \& Giménez, 2011). Por esta razón, el uso de la metodología check-all-that-apply (CATA) para identificar el perfil ideal de un producto, puede ser una alternativa para evitar los problemas del uso de escalas con consumidores (Ares et al., 2014).

Los cuestionarios CATA poseen ventajas objetivas, especialmente en relación al uso con los consumidores. La más obvia, según lo reportado en la literatura (Ares et al., 2014), es la facilidad de uso con respuestas más inmediatas a un cuestionario CATA que frente a otros métodos, especialmente los que utilizan escalas de intensidad. Otra ventaja importante es la rapidez de este método ya que, considerando los mismos atributos, se tarda aproximadamente la mitad del tiempo para evaluar un conjunto de muestras respecto al necesario cuando se utilizan escalas de intensidad. Una ventaja adicional de este método es la simplicidad del procesamiento de los datos ya que estos son esencialmente una respuesta binaria.

En la metodología CATA, se le presenta a los consumidores un listado de términos y se les pregunta acerca de cuál de estos describe mejor las características sensoriales de la muestra analizada (Meyners \& Castura, 2014). CATA ha sido reportada como una metodología fácil e intuitiva para los consumidores con la que puede obtenerse información válida y repetible (Jaeger et al., 2013; Ares et al., 2014), pero debe considerarse que, si la cantidad de términos aumenta, la sensación de facilidad puede disminuir. Por tanto, aún existen cuestiones que deben recibir una atención significativa, como la forma de seleccionar los términos que deben incluirse en las preguntas CATA y cuántos términos deben ser utilizados (Jaeger et al., 2015).

Además, las largas listas de términos pueden incluir términos focales y su antónimo (por ejemplo, "dulce" y "no dulce '), lo que podría ayudar a quien dirige la prueba para comprobar la validez de las respuestas de los consumidores. Se ha señalado como debilidad del sistema la incertidumbre sobre el significado de los términos sin marcar, ya que los participantes pueden dejar un término sin marcar porque no se aplica al producto, porque lo pasan por alto o porque se sienten neutrales o indecisos sobre su aplicabilidad del término al producto. Por tanto, el uso de los antónimos puede ser útil para determinar que significa un término sin marcar, así como identificar a aquellos consumidores que marcan tanto al término como a su antónimo para poder remover sus respuestas del análisis (Ares et al., 2014; Jaeger et al., 2015).

Una alternativa a CATA es la metodología Rate-All-That-Apply (RATA), que ha sido considerada más efectiva para discriminar entre muestras con

\section{Cumbres}


pequeñas diferencias sensoriales, con la ventaja de requerir muestras de menor tamaño (Ares et al., 2014), pero se recomienda su uso con jueces semi entrenados (Giacalone \& Hedelund, 2016).

El objetivo de este trabajo fue identificar, utilizando la metodología CATA, los atributos que podrían emplearse para la optimización posterior de formulaciones de hamburguesas con compuestos que aumenten su funcionalidad.

\section{MATERIALES Y MÉTODOS}

Formulaciones: todos los ingredientes fueron adquiridos frescos en el mercado local. La carne de cabra, proveniente de los muslos, fue conservada entre $0^{\circ} \mathrm{C}$ y $4^{\circ} \mathrm{C}$, por no más de 24 horas, hasta su utilización.

Para la formulación se estableció un diseño factorial, en el que los porcentajes de albahaca y perejil se manipularon como se muestra en la tabla 1. Se añadió $6 \%$ de huevo, 2,1\% de sal y carne caprina molida, en cantidad suficiente (84,9 - 89,9 \%). Durante la elaboración, se mezclaron los ingredientes y se amasaron en un recipiente de acero inoxidable, hasta dar la consistencia esperada y de manera uniforme se elaboraron formas circulares y planas, de aproximadamente $1 \mathrm{~cm}$ de altura x $8 \mathrm{~cm}$ de diámetro; éstas se escaldaron con vapor de agua $\left(100^{\circ} \mathrm{C}\right)$ a $15 \mathrm{~cm}$ de altura, por espacio de 10 minutos y se colocaron de forma separada en una bandeja plana de acero inoxidable y se dejaron enfriar a temperatura ambiente para preservarlas en refrigeración $\left(4^{\circ} \mathrm{C}\right)$ hasta la degustación.

Tabla 1. Diseño experimental con variaciones en los porcentajes de albahaca y perejil.

\begin{tabular}{|l|l|l|l|}
\multicolumn{5}{|c|}{ PEREJIL (\%) } \\
\hline 1,5 & 2,5 & 3,5 \\
\hline 2,5 & F1 & F4 & F7 \\
\hline 3,5 & F2 & F5 & F8 \\
\hline
\end{tabular}

Evaluación sensorial: Para la evaluación, las hamburguesas se cocinaron hasta alcanzar una temperatura interna de $70{ }^{\circ} \mathrm{C}$ y se cortaron en fracciones de aproximadamente $40 \mathrm{~g}$. Todas las muestras fueron codificadas mediante un código de tres cifras seleccionadas al azar. Se emplearon 80 consumidores potenciales del producto a los que se les presentó una boleta de evaluación para realizar el test CATA consistente en seleccionar de una lista de 13 atributos, los que les parecían adecuados para describir cada una de las nueve formulaciones.

Los atributos presentados a los jueces fueron: Dureza, Salado, Ácido, Sabor intenso, Rancio, Jugosidad, Amargo, Regusto, Consistencia, Picante, Agrio y Olor característico.

Análisis estadísticos: Se utilizó el programa IBM SPSS Statistics 22. A los datos obtenidos mediante la prueba CATA se les evaluó la confiabilidad mediante el valor alfa de Crombach y se les realizó un análisis de varianza 
para determinar las diferencias entre fórmulas y atributos. En caso de ser necesario se realizó la prueba de Rangos Múltiples de Duncan.

\section{RESULTADOS Y DISCUSIÓN}

Prueba CATA: Los cuestionarios basados en la metodología CATA han demostrado ser alternativas simples para evaluar las percepciones de los consumidores con respecto a los productos alimenticios (Ares et al., 2010). El cuestionario fue aplicado a los mismos 80 consumidores potenciales del producto que en un experimento anterior lo habían evaluado mediante una escala hedónica.

A los datos obtenidos de todos los consumidores para todas las formulaciones, se les determinó el valor del coeficiente alfa de Cronbach, el cual sirve para medir la consistencia interna o fiabilidad de una escala. La validez de un instrumento (en este caso, la escala de atributos) se refiere al grado en que el instrumento mide aquello que pretende medir. Frías (2014), resume como guías de fiabilidad según el valor del alfa de Cronbach las siguientes referencias: menor a 0,5 inaceptable, de 0,5 a 0,6 pobre; de 0,6 a 0,7 cuestionable; de 0,7 a 0,8 aceptable; de 0,8 a 0,9 bueno y mayor que 0,9 excelente. En este trabajo se obtuvo un valor de 0,81 , indicando un buen grado de consistencia interna.

El resumen de los resultados de la prueba CATA es mostrado en la Tabla 3 , donde se refieren las frecuencias con que se identificó cada atributo para cada formulación. Estos resultados fueron sometidos a un análisis de varianza con el fin de identificar los atributos que diferencian las formulaciones.

Tabla 3. Frecuencia con que se identificaron los atributos para cada formulación, valor F y significación estadística.

\begin{tabular}{|c|c|c|c|c|c|c|c|c|c|c|c|}
\hline \multirow[t]{2}{*}{ ATRIBUTOS } & \multirow[t]{2}{*}{$\mathbf{F}$} & \multirow[t]{2}{*}{ SIGN } & \multicolumn{9}{|c|}{ FRECUENCIA POR FORMULACIÓN } \\
\hline & & & F1 & $\mathrm{F} 2$ & F3 & F4 & F5 & F6 & F7 & F8 & F9 \\
\hline Dureza* & 4,668 & 0,000 & 71 & 70 & 65 & 69 & 52 & 55 & 56 & 69 & 70 \\
\hline Salado & 0,911 & 0,507 & 68 & 65 & 62 & 67 & 72 & 61 & 65 & 67 & 66 \\
\hline Ácido & 0,251 & 0,981 & 1 & 0 & 1 & 1 & 1 & 1 & 1 & 1 & 2 \\
\hline Sabor intenso* & 31,739 & 0,000 & 64 & 30 & 6 & 9 & 9 & 12 & 11 & 14 & 12 \\
\hline Rancio & 0,875 & 0,537 & 0 & 0 & 1 & 0 & 0 & 0 & 0 & 1 & 0 \\
\hline Jugosidad* & 16,912 & 0,000 & 60 & 59 & 75 & 32 & 75 & 62 & 63 & 77 & 69 \\
\hline Amargo & 1,000 & 0,435 & 0 & 0 & 0 & 0 & 0 & 0 & 1 & 0 & 0 \\
\hline Regusto* & 2,974 & 0,005 & 46 & 60 & 60 & 69 & 50 & 54 & 61 & 58 & 57 \\
\hline Consistencia* & 13,234 & 0,001 & 65 & 63 & 75 & 72 & 73 & 74 & 74 & 77 & 75 \\
\hline Picante* & 3,582 & 0,000 & 10 & 11 & 2 & 1 & 3 & 1 & 2 & 4 & 4 \\
\hline Agrio & 1,556 & 0,135 & 2 & 0 & 0 & 0 & 0 & 1 & 2 & 0 & 3 \\
\hline Olor característico & 0,293 & 0,968 & 75 & 76 & 74 & 75 & 73 & 76 & 72 & 73 & 75 \\
\hline
\end{tabular}

Los atributos marcados con asteriscos mostraron diferencias significativas en el análisis de varianza. Por tanto, ellos son los que establecen las dife- 
rencias entre las fórmulas. Estos fueron Dureza, Sabor intenso, Jugosidad, Regusto, Consistencia y Sabor Picante. Esto coincide con lo observado por otros investigadores (Panuncio et al., 2013; Ramírez-Rivera et al., 2016) quienes encontraron que factores asociados a la percepción visual, olor, sabor y textura, pueden ser empleados por consumidores para discriminar entre diferentes formulaciones de hamburguesas.

$\mathrm{Al}$ analizar los valores encontrados, se observa que la detección de sabor intenso y sabor picante fue mayor para las fórmulas F1 y F2, asociado a la menor presencia de perejil y relativamente baja presencia de albahaca. Esto puede indicar que los consumidores lo que en realidad detectaban era el sabor característico de la carne caprina, el cual en el resto de las fórmulas se enmascara parcialmente por la acción de los condimentos empleados. En todas las formulaciones, los jueces declararon la presencia de regusto, lo cual también puede asociarse a ese sabor característico. En general los jueces consideraron que los atributos dureza, consistencia y jugosidad, eran detectables en todas las formulaciones.

Los atributos que no establecen diferencias entre las muestras, también son importantes. El atributo salado siempre estuvo presente, lo cual indica que es un atributo característico de este tipo de producto. En cambio, ácido, amargo, rancio y agrio, que constituyen defectos posibles del producto, estuvieron prácticamente ausentes de los señalamientos indicando que estos defectos no fueron detectados en las formulaciones estudiadas.

La tabla 4 muestra el rango de jueces que identificaron cada uno de los atributos significativos dentro de las nueve fórmulas. En los atributos; sabor intenso, dureza, jugosidad, regusto y consistencia, la percepción de los atributos fue significativamente notable en algunas formulaciones, en cambio dureza, regusto, jugosidad y consistencia fueron mayoritariamente señalados en todas las formulaciones, sabor intenso mostró un amplio rango de respuestas, indicando que en las formulaciones más condimentadas el sabor característico de la carne caprina fue enmascarado. El atributo picante, fue importante para muy pocos jueces, con notable diferencia entre F1 y F2 en relación al resto. Cabe señalar que visto globalmente, estas son las fórmulas menos condimentadas. La tabla 5 muestra los resultados de la aplicación de la prueba de rangos múltiples de Duncan a los atributos por fórmula.

Tabla 4. Rango de mención por atributo.

\begin{tabular}{|c|c|c|c|c|c|c|}
\hline ATRIBUTO & Dureza & $\begin{array}{l}\mathrm{S} \text { a b o r } \\
\text { intenso }\end{array}$ & Jugosidad & Regusto & Consistencia & Picante \\
\hline RANGO & $52-71$ & $6-64$ & $32-77$ & $46-69$ & $63-77$ & $1-11$ \\
\hline
\end{tabular}

Al analizar los valores encontrados (Tabla 5) se observa que la detección de sabor intenso y sabor picante fue mayor para las fórmulas F1 y F2, asociado a la menor presencia de perejil y relativamente baja presencia de albahaca. Esto puede indicar que los consumidores en realidad detectaban el sabor característico de la carne caprina, el cual en el resto de las fórmulas se enmascara parcialmente por la acción de los condimentos empleados. En 
todas las formulaciones, los jueces, de forma mayoritaria, declararon la presencia de regusto, lo cual también puede asociarse a ese sabor característico. En general los jueces consideraron que los atributos dureza, consistencia y jugosidad, eran detectables en todas las formulaciones.

Tabla 5. Prueba de rangos múltiples de Duncan a los atributos por fórmula

\begin{tabular}{|l|l|l|l|l|l|l|l|l|l|}
\hline FÓRMULA & F1 & F2 & F3 & F4 & F5 & F6 & F7 & F8 & F9 \\
\hline ALBAHACA \% & 1.50 & 2.50 & 3.50 & 1.50 & 2.50 & 3.50 & 1.50 & 2.50 & 3.50 \\
\hline PEREJIL \% & 1.50 & 1.50 & 1.50 & 2.50 & 2.50 & 2.50 & 3.50 & 3.50 & 3.50 \\
\hline DUREZA & $71 \mathrm{~b}$ & $70 \mathrm{~b}$ & $65 \mathrm{~b}$ & $69 \mathrm{~b}$ & $52 \mathrm{a}$ & $55 \mathrm{a}$ & $56 \mathrm{a}$ & $69 \mathrm{~b}$ & $70 \mathrm{~b}$ \\
\hline SABOR INTENSO & $64 \mathrm{c}$ & $30 \mathrm{~b}$ & $6 \mathrm{a}$ & $9 \mathrm{a}$ & $9 \mathrm{a}$ & $12 \mathrm{a}$ & $11 \mathrm{a}$ & $14 \mathrm{a}$ & $12 \mathrm{a}$ \\
\hline JUGOSIDAD & $60 \mathrm{~b}$ & $59 \mathrm{~b}$ & $75 \mathrm{c}$ & $32 \mathrm{a}$ & $75 \mathrm{c}$ & $62 \mathrm{~b}$ & $63 \mathrm{~b}$ & $77 \mathrm{c}$ & $69 \mathrm{bc}$ \\
\hline REGUSTO & $46 \mathrm{a}$ & $60 \mathrm{bc}$ & $60 \mathrm{bc}$ & $69 \mathrm{c}$ & $50 \mathrm{ab}$ & $54 \mathrm{ab}$ & $61 \mathrm{bc}$ & 5 & $57 \mathrm{abc}$ \\
\hline CONSISTENCIA & $65 \mathrm{ab}$ & $63 \mathrm{a}$ & $75 \mathrm{c}$ & $72 \mathrm{bc}$ & $73 \mathrm{c}$ & $74 \mathrm{c}$ & $74 \mathrm{c}$ & $77 \mathrm{c}$ & $75 \mathrm{c}$ \\
\hline PICANTE & $10 \mathrm{~b}$ & $11 \mathrm{~b}$ & $2 \mathrm{a}$ & $1 \mathrm{a}$ & $3 \mathrm{a}$ & $1 \mathrm{a}$ & $2 \mathrm{a}$ & $4 \mathrm{a}$ & $4 \mathrm{a}$
\end{tabular}

Cantidades con letras diferentes en una misma fila, difieren significativamente entre sí $(p<0,05)$.entre sí $(\mathrm{p}<0,05)$.

\section{CONCLUSIONES}

Los atributos dureza, sabor intenso, jugosidad, regusto, consistencia y sabor picante son los que los consumidores detectan como diferenciantes entre las formulaciones estudiadas, estando los atributos sabor intenso y sabor picante asociados fundamentalmente a las hamburguesas menos condimentadas.

\section{REFERENCIAS BIBLIOGRÁFICAS}

Aguilar, P. V., Castillo, C., \& Gutowski, L. (2014). Metodología para diseñar un producto alimenticio por medio de la identificación de los factores que influyen en la decisión de compra. Revista Venezolana de Ciencia y Tecnología de Alimentos, 5(1), 018-030.

Alegre, Y., de la Fuente, A., Orte, P. H., González, V. F., \& Navajas, M. P. S. (2015). Aplicación de nuevas técnicas rápidas de análisis sensorial a la investigación enológica: prueba de categorización de la calidad seguida de un perfilado rápido. ACE: Revista de enología, (152), 2.

Antúnez, L., Vidal, L., de Saldamando, L., Giménez, A., \& Ares, G. (2016). Comparison of consumer-based methodologies for sensory characterization: Case study with four sample sets of powdered drinks. Food Quality and Preference.

Ares, G., Antúnez, L., Giménez, A., Roigard, C. M., Pineau, B., Hunter, D. C., $\&$ Jaeger, S. R. (2014). Further investigations into the reproducibility of check-all-that-apply (CATA) questions for sensory product characterization elicited by consumers. Food Quality and Preference, 36, 111-121.

Ares, G., Barreiro, C., Deliza, R., Giménez, A., \& Gámbaro, A. (2010). Applica- 
tion of a Check-All-That-Apply Question to the Development of Chocolate Milk Desserts. Journal of Sensory Studies, 25(1), 67-86.

Ares, G., Bruzzone, F., \& Gimenez, A. (2011). Is a consumer panel able to reliably evaluate the texture of dairy desserts using unstructured intensity scales? Evaluation of global and individual performance. Journal of Sensory Studies, 26(5), 363-370

Bruzzone, F., Vidal, L., Antúnez, L., Giménez, A., Deliza, R., \& Ares, G. (2015). Comparison of intensity scales and CATA questions in new product development: Sensory characterization and directions for product reformulation of milk desserts. Food Quality and Preference, 44, 183-193.

Grunert, K. G., \& van Trijp, H. C. M. (2014). Consumer-Oriented New Product Development. Encyclopedia of Agriculture and Food Systems, 2, 375-386.

Giacalone, D. (2015). Questionari check-all-that-apply (CATA): aspetti metodologici ed esempi applicativi nell'ambito della sensory \& consumer science. Atti Del V Convegno Nazionale Della Società Italiana Di Scienze Sensoriali.

Giacalone, D., \& Hedelund, P. I. (2016). Rate-all-that-apply (RATA) with semi-trained assessors: An investigation of the method reproducibility at assessor-, attribute-and panel-level. Food Quality and Preference, 51, 65-71.

Heymann, H., King, E. S., \& Hopfer, H. (2014). Classical descriptive analysis. In Novel techniques in sensory characterization and consumer profiling (pp. 9-40). CRC Press.

Jaeger, S., Chheang, S. L., Christina, M. B., Giménez, A., Vidal, L., \& Ares, G. (2013). Check-all-that-apply (CATA) responses elicited by consumers: Within-assessor reproducibility and stability of sensory product characterizations. Food Quality and Preference, 30, 56-67.

Jaeger, S. R., Beresford, M. K., Paisley, A. G., Antúnez, L., Vidal, L., Cadena, R. S., ... \& Ares, G. (2015). Check-all-that-apply (CATA) questions for sensory product characterization by consumers: Investigations into the number of terms used in CATA questions. Food Quality and Preference, 42, 154-164.

Lawless, H. T., \& Heymann, H. (2010). Sensory evaluation of food. Principles and practices (2nd ed.) New York: Springer

Meyners, M., \& Castura, J. C. (2014). Check-all-that-apply questions. In P. Varela \& G. Ares (Eds.), Novel techniques in sensory characterization and consumer profiling (pp. 271-305). Boca Raton: CRC Press.

Moskowitz, H., \& Hartmann, J. (2008). Consumer research: creating a solid base for innovative strategies. Trends in Food Science \& Technology, 19(11), 581-589.

Panuncio, A., Cardeza, L., Quintero, M., Solé, M. L., Barrios, S., \& Gámbaro, A. (2013). Efecto de la incorporación de transglutaminasa microbiana en las propiedades sensoriales de hamburguesas de desmenuzado de merluza (Merluccius hubbsi). Innotec, (8).

Ramírez-Rivera, E. D. J., Juárez-Barrientos, J. M., Rodríguez-Miranda, J., Ramírez-García, S. A., Villa-Ruano, N., \& Ramón-Canul, L. G. (2016). Comparación de mapas de preferencia mediante el análisis descriptivo cuantitativo y perfil flash en hamburguesas. Ecosistemas y recursos agropecuarios, 3(7), 103-112. 
Worch, T., \& Punter, P. (2014). Ideal profiling. In P. Varela \& G. Ares (Eds.), Novel techniques in sensory characterization and consumer profiling (pp. 85136). Boca Raton: CRC Press.

Worch, T., Lê, S., Punter, P., \& Pagès, J. (2012). Extension of the consistency of the data obtained with the Ideal Profile Method: Would the ideal products be more liked than the tested products?. Food quality and preference, 26(1), 74-80.

\section{Cumbres}

